\title{
Osteoartritis: cuantificación del ligando del receptor nuclear kappa- $\beta$ (RANKL) en líquido sinovial de equinos Fina Sangre de Carrera
}

\author{
Osteoarthritis: quantification of the receptor activator of nuclear factor \\ kappa- $\beta$ ligand (RANKL) in synovial fluid of Thoroughbred horses
}

\author{
Luis De Negri ${ }^{1,4}$, Luis Olguín', Leonardo Pavez ${ }^{2,3}$
}

\section{Resumen}

\begin{abstract}
Osteoartritis (OA) es una enfermedad de gran impacto que produce invalidez funcional a temprana edad en los caballos de carrera donde su diagnóstico generalmente se lleva a cabo en los estados avanzados de la enfermedad. El objetivo de esta investigación fue demostrar la presencia del ligando del receptor activador del factor nuclear kappa- $\beta$ (RANKL) en líquido sinovial de equinos en training. Se evaluó clínica y radiográficamente a tres caballos sanos de dos años y cuatro caballos de 3-4 años con signos de inflamación articular. Se les extrajo líquido sinovial para cuantificar la expresión de RANKL en diferentes estados patológicos del carpo mediante una prueba de ELISA. El valor promedio de RANKL en los caballos sanos fue de $33.13 \pm 8.30 \mathrm{pg} / \mathrm{ml}$ y en los caballos con signos de OA de $49.03 \pm 5.95 \mathrm{pg} / \mathrm{ml}(\mathrm{p}<0.05)$.
\end{abstract}

Palabras clave: osteoartritis; equinos; RANKL; RANK; NFk $\beta$; osteoclastogénesis

\section{AbSTRaCt}

Osteoarthritis (OA) is a disease of great impact that produces functional disability at an early age in racehorses where its diagnosis is usually carried out in advanced stages

\footnotetext{
${ }^{1}$ Escuela de Medicina Veterinaria, Facultad de Medicina Veterinaria y Agronomía, Universidad de las Américas, Santiago, Chile

${ }^{2}$ Instituto de Ciencias Naturales, Universidad de las Américas, Santiago, Chile

${ }^{3}$ Departamento de Ciencias Químicas y Biológicas, Universidad Bernardo O'Higgins, Santiago, Chile

${ }^{4}$ E-Mail: dr.luisdenegri@gmail.com
}

Financiamiento: Fondo concursable proyectos de investigación. Dirección de Investigación. Vicerrectoría Académica. $N^{\circ}$ PI-015-2016

Recibido: 20 de agosto de 2018

Aceptado para publicación: 21 de febrero de 2019 
of the disease. The aim of this study was to demonstrate the presence of the activating receptor ligand of nuclear factor kappa- $\beta$ (RANKL) in synovial fluid of equines in training. Three healthy horses of two years and four horses of 3-4 years of age with signs of joint inflammation were evaluated clinically and radiographically. Synovial fluid was extracted to quantify the expression of RANKL in different pathological states of the carpus by an ELISA test. The average value of RANKL in healthy horses was $33.13 \pm 8.30 \mathrm{pg} / \mathrm{ml}$ and in horses with OA signs of $49.03 \pm 5.95 \mathrm{pg} / \mathrm{ml}(\mathrm{p}<0.05)$.

Key words: osteoarthritis; equine; RANKL; RANK; NF-k $\beta$; osteoclastogenesis

\section{INTRODUCCIÓN}

En equinos de deporte, la principal causa de invalidez funcional son las afecciones del sistema músculo esquelético (Martig et al., 2014), donde la osteoartritis (OA) es una de las artropatías más frecuentes (Lacourt et al., 2012; Caggiano et al., 2013; Polli et al., 2013), que se traduce en claudicación (Reed et al., 2012) y pérdidas en días de entrenamiento; situación que implica, además, altos costos en su tratamiento (Oke y McIlwraith, 2010).

Los signos clínicos de la OA son característicos (Ross et al., 2012; Seidel et al., 2013), entre ellos, aumento de volumen articular, inflamación de tejidos blandos, sinovitis y capsulitis (De Negri, 2006), calor local, movimientos articulares limitados (en los estados avanzados de la enfermedad) y diferentes grados de claudicación (Mcllwraith, 2009). No se sabe si se genera en la cápsula articular, la sinovia, ligamentos, periostio o el hueso, dado que todos estos tienen nervios sensitivos que responden a la distención y mediadores químicos como quininas y neuropéptidos (sustancia $\mathrm{P}$ ), los que junto con mediadores como interleuquina $1 \mathrm{y}$ prostaglandina $\mathrm{E}_{2}\left(\mathrm{IL}-1\right.$ y $\mathrm{PGE}_{2}$ ) sensibilizan a las fibras para ser más reactivas después de una estimulación mecánica (Frisbie, 2012).

Los principales cambios radiográficos en ejemplares con OA son: osteofitos periarticulares, aumento de la densidad ósea subcondral con ocasional lisis, lesiones quís- ticas y, en los estados terminales, disminución del espacio articular o anquilosis (Lacourt et al., 2012), así como entesofitos en las zonas de inserción de la cápsula articular y mineralización de tejidos blandos (Frisbie et al., 2015). En este contexto, la identificación precoz de la OA puede ser crítica, tanto en su tratamiento como en la prevención de la progresión de la enfermedad (Kamm et al., 2013), y dado que su diagnóstico se basa en exámenes radiográficos, se tiene el inconveniente de que en estados tempranos tienen una baja sensibilidad para detectar cambios en los tejidos blandos y hueso subcondral (Mcllwraith, 2009), por lo que las lesiones son diagnosticadas cuando ya se ha producido un daño articular significativo (Mcllwraith et al., 2012).

Se requiere comprender la contribución del micro daño provocado por las cargas repetitivas en casos de enfermedad osteocondral en la superficie articular (Peat y Kawcak, 2015; Bani Hassan et al., 2016; Malekipour et al., 2016), como ocurre en el humano donde se ha logrado un mayor entendimiento de la enfermedad a través de los mecanismos moleculares que producen osteoartritis donde desórdenes del sistema RANKL/RANK/OPG están asociados a enfermedades como osteoporosis, artritis reumatoide y tumores óseos (Liu y Zhang, 2015). Por otra parte, la transferencia de solutos a través del cartílago articular y plato del hueso subcondral, podría contribuir a entender los mecanismos de la progresión de la $\mathrm{OA}$, lo cual se viene estudiando en humanos y equinos mediante microtomografía 
computarizada (Arbabi et al., 2016; Bertugglia et al., 2016).

Es así que en la generación de lesiones en el hueso subcondral, se puede identificar dos factores producidos por los osteoblastos que tienen un rol crucial en la regulación de la resorción ósea por osteoclastos, uno es RANKL y el otro es osteoprotegerina (OPG), siendo estos una de las mayores fuentes que controlan la resorción ósea (Zupan et al., 2013). RANKL es una proteína de membrana tipo II, producida por macrófagos, neutrófilos, queratinocitos, células endoteliales y fibroblastos. Es considerada la citoquina clave que induce osteoclastogénesis (Nakashima et al., 2012), que promueve la proliferación celular y que contribuye directamente en el proceso de destrucción ósea (Okamoto y Takanayagi, 2011; Maxhimer et al., 2015). RANKL también permite la maduración, diferenciación y activación de osteoclastos mediante la unión a su agonista RANK (Ariyoshi et al., 2014). Está presente en la superficie de pre-osteoclastos, que traduce sus señales reclutando moléculas adaptadoras, como el factor asociado al receptor TNF (TRAF), donde TRAF-6 es capaz de estimular la osteoclastogénesis (Nakashima et al., 2012), permitiendo la translocación del factor de transcripción NF$\mathrm{k} \beta$ al núcleo (Boyce et al., 2015), que controla la respuesta inflamatoria e inmunológica.

Una vez finalizados los procesos de señalización que indujeron la osteoclastogénesis gatillada por RANKL, los osteoclastos maduros secretan protones, iones cloruro y colagenasas, bajo la formación en cepillo de la membrana celular que enfrenta la superficie del hueso a reabsorber. El ácido clorhídrico se forma bajo la membrana y disuelve el componente mineral del hueso y la catepsina $\mathrm{K}$ es secretada para degradar la matriz durante la progresión de la OA (Boyce, 2013; Bertuglia et al., 2016). Así, finalmente los osteoclastos se mueven a lo largo de las superficies del hueso, aumentando las lagunas de resorción hasta que el proceso se haya completado (Boyce et al., 2015).
En este contexto, el objetivo de este estudio fue demostrar la presencia del ligando del receptor activador del factor nuclear kappa- $\beta$ (RANKL) en líquido sinovial de equinos en entrenamiento, el cual contribuye directamente en el proceso de destrucción ósea estimulando la osteoclastogénesis en la OA.

\section{Materiales y Métodos}

Se utilizaron siete equinos Fina Sangre de Carrera, que fueron sometidos a examen clínico, radiográfico y de líquido sinovial de la articulación intercarpal. De estos, tres tenían dos años, eran clínicamente sanos y no presentaban signos de sinovitis, dolor articular o claudicación. Los otros cuatro ejemplares ( 2 de 3 años y 2 de 4 años) presentaban diversas manifestaciones de OA, caracterizada a través de tres signos clínicos:

- Sinovitis leve, cuando la efusión sinovial solo era evidente a la palpación, sinovitis moderada cuando era observable a la inspección y la efusión era fluctuante a la palpación, y sinovitis marcada al ser evidente a la inspección con efusión dura a la palpación.

- Dolor a la flexión marcada, cuando al flexionar la articulación carpal se presenta renuencia al procedimiento clínico.

- Claudicación leve es aquella difícil de observar al paso o trote; y claudicación moderada la observable al trote en toda circunstancia.

En el examen radiográfico de los ejemplares se utilizaron las vistas convencionales dorsolateral-palmaromedial oblicua (DLPMO), dorsomedial-palmarolateral oblicua (DMPLO), latero-medial (LM) y lateromedial flectada (LMF). Se empleó un equipo radiográfico MinXray TR90. La extracción del líquido sinovial $(1 \mathrm{ml})$ se realizó en la articulación intercarpal mediante artrocentesis para detectar y cuantificar RANKL con un kit comercial de ELISA (Horse receptor activator of nuclear factor kappa B ligand [RANKL] ELISA Kit, MyBioSource Cat. 
MBS019968), siguiendo los procedimientos sugeridos por el fabricante. Las muestras fueron refrigeradas y llevadas al laboratorio en un lapso de 30 minutos, donde fueron centrifugadas a $3000 \mathrm{rpm}$ por $20 \mathrm{~min}$ y guardadas a $-80{ }^{\circ} \mathrm{C}$ hasta su procesamiento.

Se utilizó la prueba de Shapiro Wilk para determinar si los valores de RANKL se distribuyen de forma normal y la prueba t-Student para determinar posibles diferencias estadísticas de los valores de RANKL entre caballos sanos y con OA.

Los procedimientos empleados fueron suscritos a la «Declaración de Singapur sobre Integridad en la Investigación»y y revisados por el Comité de Ética de la Universidad de las Américas.

\section{Resultados}

Los valores de RANKL contenidos en el líquido sinovial de los equinos sanos y con signos de OA se presentan en los Cuadros 1 y 2. Los caballos clínicamente sanos presentaron un factor RANKL promedio de 33.13 $\pm 8.30 \mathrm{pg} / \mathrm{ml}$ en el líquido sinovial, no presentaron signos de sinovitis, dolor a la flexión de carpo o claudicación y en el examen radiográfico no se observaron signos de lesiones congruentes con OA.

Los caballos con signos clínicos de inflamación articular y radiográficos de $\mathrm{OA}$ presentaron un factor RANKL promedio de $49.03 \pm 5.95 \mathrm{pg} / \mathrm{ml}$ en el líquido sinovial. Estos ejemplares presentaron signos de sinovitis, dolor a la flexión de carpo o claudicación y en el examen radiográfico se observaron signos de OA consistentes en osteólisis y entesofitosis en la inserción capsular. La mayoría presentó fracturas osteocondrales antiguas. Los caballos de cuatro años presentaron los mayores niveles de RANKL.

Los valores de RANKL siguieron una distribución normal $(\mathrm{p}=0.803)$. Se encontró diferencia significativa $(p<0.031)$ entre los valores de RANKL en caballos clínicamente sanos versus aquellos con signos clínicos de inflamación y radiográficos de OA.

\section{Discusión}

El presente estudio demostró la presencia de niveles de RANKL detectables en el líquido sinovial de la articulación intercarpal de equinos Fina Sangre de Carrera en entrenamiento. Estos niveles fueron significativamente más bajos en equinos de dos años sanos en comparación con aquellos de mayor edad y que presentaban diferentes grados de daño articular y congruentes con osteoartritis.

Estos resultados podrían complementarse con estudios post mortem realizados en caballos de carrera con OA postraumática, los cuales presentaban mayor densidad de osteoclastos en el hueso subcondral, lo que se correlaciona positivamente con la degeneración del cartílago y el valor (score) de RANKL en el cartílago articular (Bertuglia et al., 2016). Estos resultados podrían explicar la interrogante del compromiso del hueso subcondral a partir de una inflamación intraarticular, dada la cercana asociación física entre el hueso subcondral y cartílago, sugiriendo la posibilidad de una conversación bioquímica y molecular a través de la interfase. Es decir, esta comunicación entre la capa de cartílago calcificado y plato del hueso subcondral se traduciría en un aumento de canales vasculares comunicantes, fisuras y microfracturas que podrían transportar moléculas y solutos, tal como lo han sugerido Yuan et al. (2004) y Arbabi et al. (2016).

Por otro lado, humanos sin alteraciones óseas degenerativas evidencian niveles de RANKL sanguíneo basal de 3.6 y 4.6 pg/ml para mujeres y hombres, respectivamente (Kerschan-Schindl et al., 2008). A su vez, Fadda et al. (2015) en pacientes humanos con artritis reumatoidea observaron niveles sanguíneos de RANKL más altos que en pa- 
Cuadro 1. Niveles del receptor activador del factor nuclear kappa- $\beta$ (RANKL) en el líquido sinovial de equinos sanos

\begin{tabular}{|c|c|c|c|c|}
\hline Equino & $\begin{array}{c}\text { Edad } \\
\text { (años) }\end{array}$ & Examen clínico & Examen radiográfico & $\begin{array}{c}\text { Concentración } \\
\mathrm{pg} / \mathrm{ml})\end{array}$ \\
\hline 1 & 2 & Normal & Carpo izquierdo normal & 39.88 \\
\hline 2 & 2 & Normal & Carpo izquierdo normal & 35.72 \\
\hline 3 & 2 & Normal & Carpo derecho normal & 23.88 \\
\hline \multicolumn{3}{|c|}{ Promedio } & & $33.13 \pm 8.30$ \\
\hline
\end{tabular}

Cuadro 2. Niveles del receptor activador del factor nuclear kappa- $\beta$ (RANKL) en el líquido sinovial de equinos con signos de osteoartritis

\begin{tabular}{|c|c|c|c|c|}
\hline Equino & $\begin{array}{l}\text { Edad } \\
\text { (años) }\end{array}$ & Examen clínico & Examen radiográfico & $\begin{array}{c}\text { Concentración } \\
\mathrm{pg} / \mathrm{ml})\end{array}$ \\
\hline 4 & 4 & $\begin{array}{l}\text { Sinovitis marcada, } \\
\text { dolor leve a la flexión, } \\
\text { y claudicación leve }\end{array}$ & $\begin{array}{l}\text { Carpo izquierdo: fractura } \\
\text { carporadial borde dorso distal. } \\
\text { Osteólisis }\end{array}$ & 54.49 \\
\hline 5 & 4 & $\begin{array}{l}\text { Sinovitis marcada, } \\
\text { dolor moderado a la } \\
\text { flexión y claudicación } \\
\text { moderada }\end{array}$ & $\begin{array}{l}\text { Carpo izquierdo: entesofitosis } \\
\text { inserción cápsula hueso } \\
\text { carporadial }\end{array}$ & 51.42 \\
\hline 6 & 3 & $\begin{array}{l}\text { Sinovitis y dolor } \\
\text { marcado a la flexión y } \\
\text { claudicación leve }\end{array}$ & $\begin{array}{l}\text { Carpo derecho: fractura carpo } \\
\text { intermedio borde dorso distal y } \\
\text { osteólisis }\end{array}$ & 49.57 \\
\hline 7 & 3 & $\begin{array}{l}\text { Sinovitis y dolor } \\
\text { marcado a la flexión y } \\
\text { claudicación leve }\end{array}$ & $\begin{array}{l}\text { Carpo izquierdo: fractura } \\
\text { carporadial, osteólisis, } \\
\text { entesofitosis inserción cápsula } \\
\text { hueso carpo radial, osteofito } \\
\text { carpo intermedio borde dorso } \\
\text { distal }\end{array}$ & 40.65 \\
\hline \multicolumn{4}{|c|}{ Promedio } & $49.03 \pm 5.95$ \\
\hline
\end{tabular}

cientes control ( 4.7 vs. $3.0 \mathrm{pg} / \mathrm{ml})$. Estos valores son más bajos que los encontrados en el presente estudio, debido posiblemente a que se obtuvieron desde sangre y no desde líquido sinovial. En futuros estudios se podría medir el nivel de RANKL en sangre y líquido sinovial para evidenciar potenciales correlaciones en este indicador de daño tisular.
La evidencia sugiere que la activación de RANKL es el principal disparador de la osteoclastogénesis y la cadena de activación de factores de transcripción TRAP, CATK e integrina $\beta 3$ por la quinasa NF-K $\beta$ (Zupan et $a l ., 2013)$. Estas a su vez están asociadas al pH del medio (Bernhardt et al., 2017) y a la presencia de moléculas asociadas a 
patógenos (PAMPs) y lipopolisacáridos (LPS) (Morita et al., 2016), respectivamente, que inducen la resorción ósea y la formación de osteoclastos. Los resultados de este trabajo indican que esta situación también estaría ocurriendo en equinos de carrera.

Este es el primer estudio en detectar RANKL en el líquido sinovial de equinos Fina Sangre de Carrera en training in vivo, citoquina asociada a osteoclastogénesis (Nakashima et al., 2012) y que promueve la proliferación celular y destrucción ósea (Okamoto y Takanayagi, 2011; Maxhimer et al., 2015). Asimismo, este estudio demuestra la utilidad de la medición de esta proteína como un biomarcador en etapas tempranas de osteoartritis. Su determinación permitiría evaluar los tratamientos instaurados, tal como se ha evidenciado en estudios en humanos (Chung et al., 2014). Por otro lado, los valores elevados de RANKL en el líquido sinovial de los ejemplares estudiados podría reflejar el nivel de daño articular, permitiendo aproximaciones integrales para el manejo preventivo y control más adecuado de la osteoartritis. Las próximas investigaciones deberían comprobar estos resultados con un número mayor de animales y en diferentes etapas de osteoartritis.

\section{Conclusiones}

El presente estudio permitió identificar la presencia de niveles bajos de RANKL en líquido sinovial de la articulación intercarpal de equinos sanos y valores altos en equinos con signos de osteoartritis.

\section{Literatura Citada}

1. Arbabi V, Pouran B, Weinans $H$, Zadpoor AA. 2016. Neutral solute transport across osteochondral interface: a finite element approach. J Biomech 49: 3833-3839. doi: 10.1016/j.jbiomech.2016.10 .015
2. Ariyoshi W, Okinaga T, Knudson C, Knudson W, Nishihara T. 2014. High molecular weight hyaluronic acid regulates osteoclast formation by inhibiting receptor activator of NF-kB ligand through Rho kinase. Osteoarthr Cartilage 22: 111-120. doi: 10.1016/ j.joca.2013.10.013

3. Bani Hassan E, Mirams M, GhasemZadeh A, Mackie EJ. 2016. Role of subchondral bone remodelling in collapse of the articular surface of thoroughbred racehorses with palmar osteochondral disease. Equine Vet J 48: 228-233. doi: 10.1111/evj.12415

4. Bernhardt A, Koperski K, Schumacher M, Gelinsky M. 2017. Relevance of osteoclastspecific enzyme activities in cell-based in vitro resorption assays. Eur Cells Mater 33: 28-42. doi: 10.22203/eCM.v033a03

5. Bertuglia A, Lacourt M, Girard C, Beauchamp G, Richard H, Laverty $S$. 2016. Osteoclasts are recruited to the subchondral bone in naturally occurring post-traumatic equine carpal osteoarthritis and may contribute to cartilage degradation. Osteoarthritis Cartilage 24: 555-566. doi: 10.1016/j.joca.2015.10.008

6. Boyce BF. 2013. Advances in osteoclast biology reveal potential new drug targets and new roles for osteoclasts. J Bone Miner Res 28: 711-722. doi: 10.1002/ jbmr.1885

7. Воусе B, Xiu Y, Li j, Xing L, Yao Z. 2015. NF-KB-mediated regulation of osteoclastogenesis. Endocrinol Metab 30: 35-44. doi: 10.3803/EnM.2015.30.1.35

8. Caggiano N, Rolando J, Polli M, Perrone, Marino M, De Simone E, Chiappe A. 2013. Citokines, metalloproteinases and bisphosphonates: control keys for degenerative joint disease in horses. REDVET 14(7). [Internet]. Disponible en: http://www.veterinaria.org/ revistas/redvet $/ \mathrm{n} 070713 / 071305$.pdf

9. Chung PL, Zhou S, Eslami B, Shen L, LeBoff MS, Glowacki J. 2014. Effect of age on regulation of human osteoclast differentiation. J Cell Biochem 115: 1412-1419. doi: 10.1002/jcb.2479 
10. De Negri L. 2006. Manual de referencia en clínica de equinos. Santiago: Ed Universidad Mayor. $\mathrm{p}$ 21-22.

11. Fadda S, Hamdy A, Abulkhair E, Mahmoud Elsify H, Mostafa A. 2015. Serum levels of osteoprotegerin and RANKL in patients with rheumatoid arthritis and their relation to bone mineral density and disease activity. Egyptian Rheumatologist 37: 1-6. doi: 10.1016/ j.ejr.2014.06.001

12. Frisbie D. 2012. Synovial joint biology and pathobiology. In: Auer JA, Stick JA (eds). Equine surgery. $4^{\text {th }}$ ed. WB Saunders. p 1096-1114.

13. Frisbie DD, McCarthy HE, Archer CW, Barrett MF, McIlwraith CW. 2015. Evaluation of articular cartilage progenitor cells for the repair of articular defects in an equine model. J Bone Joint Surg Am 97: 484-493. doi: 10.2106/ JBJS.N.00404

14. Kamm JL, Frisbie DD, McIlwraith CW, Orr KE. 2013. Gene biomarkers in peripheral white blood cells of horses with experimentally induced osteoarthritis. Am J Vet Res 74: 115-121. doi: 10.2460/ajvr.74.1.115

15. Kerschan-Schindl K, Wendlova J, Kudlacek S, Gleis A, Woloszczuk W, Pietschmann P. 2008. Serum levels of receptor activator of nuclear factor $\mathrm{kB}$ ligand (RANKL) in healthy women and men. Exp Clin Endocrinol Diabetes 116: 491-495. doi: 10.1055/s-2007-993142

16. Lacourt M, Gao C, Li A, Girard C, Beauchamp G, Henderson JE, Laverty S. 2012. Relationship between cartilage and subchondral bone lesions in repetitive impact trauma-induced equine osteoarthritis. Osteoarthritis Cartilage 20: 572-583. doi: 10.1016/ j.joca.2012.02.004

17. Liu Wei, Zhang X. 2015. Receptor activator of nuclear factor $\mathrm{KB}$ ligand (RANKL)/RANK/osteoprotegerin system in bone and other tissues (review). Mol Med Rep 11: 3212-3218. doi: 10.3892/mmr.2015.3152
18. Malekipour F, Oetomo D, Lee PV. 2016. Equine subchondral bone failure threshold under impact compression applied through articular cartilage. J Biomech 49: 2053-2059. doi: 10.1016/ j.jbiomech.2016.05.016

19. Martig S, Chen W, Lee PV, Whitton RC. 2014. Bone fatigue and its implications for injuries in racehorses. Equine Vet J 46: 408-415. doi: 10.1111/ evj.12241

20. Maxhimer JB, Bradley JP, Lee JC. 2015. Signaling pathways in osteogenesis and osteoclastogenesis: lessons from cranial sutures and applications to regenerative medicine. Genes Dis 2: 57 68. doi: 10.1016/j.gendis.2014.12.004

21. McIlwraith CW, Frisbie DD, Kawcak CE. 2012. The horse as a model of naturally occurring osteoarthritis. Bone Joint Res 1: 297-309. doi: 10.1302/20463758.111.2000132

22. McIlwraith CW. 2009. Advances in the diagnosis of joint disease. In: Proc $11^{\text {th }}$ International Congress of World Equine Veterinary Association. Guarujá, SP, Brazil.

23. Morita M, Yoshida S, Iwasaki R, Yasui T, Sato Y, Kobayashi T, Watanabe R, et al. 2016. Smad 4 is required to inhibit osteoclastogenesis and maintain bone mass. Sci Rep 6: 35221. doi: 10.1038/ srep35221

24. Nakashima T, Hayashi M, Takayanagi H. 2012. New insights into osteoclastogenic signaling mechanisms. Trends Endocrin Met 23: 582-590. doi: 10.1016/j.tem.2012.05.005

25. Okamoto K, Takayanagi H. 2011. Regulation of bone by the adaptive immune system in arthritis. Arthritis Res Ther 13: 219. doi: 10.1186/ar3323

26. Oke S, McIlwraith CW. 2010. Review of the economic impact of osteoarthritis and oral joint-health supplements in horses. PAnnu Conv Am Equin 56: 12-16.

27. Peat FJ, Kawcak CE. 2015. Musculoskeletal oathology. Vet Clin N Am-Equine 31: 407-424. doi: 10.1016/ j.cveq.2015.04.003 
28. Polli M, Caggiano N, Rolando J, Perrone G, Marino M, De Simone E, Chiappe B. 2013. Variación del nivel de citoquinas en líquido sinovial de equinos con enfermedad articular tratados con bisfosfonatos. Rev Complut Cienc Vet 7: 6983.

29. Reed SR, Jackson BF, McIlwraith CW, Wright IM, Pilsworth R, Knapp S, Wood JL, et al. 2012. Descriptive epidemiology of joint injuries in Thoroughbred racehorses in training. Equine Vet J 44: 13-19. doi: 10.1111/ j.2042-3306.2010.00352.x

30. Ross TN, Kisiday JD, Hess T, McIlwraith CW. 2012. Evaluation of the inflammatory response in experimentally induced synovitis in the horse: a comparison of recombinant equine interleukin 1 beta and lipopolysaccharide.
Osteoarthr Cartilage 20: 1583-1590. doi: 10.1016/j.joca.2012.08.008

31. Seidel MF, Wise BL, Lane NE. 2013. Nerve growth factor. An update on the science and therapy. Osteoarthritis Cartilage 21: 1223-1228. doi: 10.1016/ j.joca.2013.06.004

32. Yuan XL, Meng HY, Wang YC, Peng J, Guo QY, Wang AY, Lu SB. 2014. Bone-cartilage interface crosstalk in osteoarthritis: potential pathways and future therapeutic strategies Osteoarthritis Cartilage 22: 1077-1089. doi: 10.1016/j.joca.2014.05.023

33. Zupan J, Jeras M, Marc J. 2013. Osteoimmunology and the influence of pro-inflammatory cytokines on osteoclasts. Biochem Medica 23: 43-63. doi: 10.11613/BM.2013.007 